\title{
Evolución atípica de cáncer de colon de larga duración
}

\author{
Atypical course of long-term colon cancer
}

Patricia Cruz-Castellanos ${ }^{1 *}$, Laura Gutiérrez-Sainz', David López², M. Isabel Esteban ${ }^{3}$ y Francisco J. de Castro ${ }^{1}$

${ }^{1}$ Servicio de Oncología Médica, Hospital Universitario La Paz, Madrid; ${ }^{2}$ Servicio de Urología, Hospital Universitario de Ciudad Real, Ciudad Real; ${ }^{3}$ Servicio de Anatomía Patológica, Hospital Universitario La Paz, Madrid. España

\section{Resumen}

Antecedentes: Las metástasis tiroideas son tremendamente infrecuentes, situándose su incidencia en torno al 1-3\% según los diferentes datos. El cáncer de colon es el segundo tumor que con más frecuencia produce metástasis en este órgano. Caso clínico: Se expone un caso de cáncer de colon de larga evolución con patrón metastásico atípico y aparición de metástasis tiroidea. Además, se señala la importancia del manejo multidisciplinario de esta patología y la necesidad emergente de establecer paneles de secuenciación masiva para una mejor caracterización molecular.

Palabras clave: Cáncer de colon. Metástasis tiroidea. Panel de secuenciación masiva.

\section{Abstract}

Background: Thyroid metastases are extremely rare, with an incidence of 1-3\% according to different data. Colon cancer is the second most common tumor that metastasizes to this organ. Case report: We report a case of a long-standing colon cancer with an atypical metastatic pattern and the appearance of a thyroid metastases. In addition, we highlight the importance of multidisciplinary management of this pathology and the emerging need to establish next generation sequencing panels for better molecular characterization.

Key words: Colon cancer. Thyroid metastases. Next generation sequencing.

\section{Introducción}

El cáncer de colon es uno de los tumores más incidentes y prevalentes en la actualidad. En todo el mundo, es el tercer tumor más frecuente en hombres y el segundo en mujeres, con una incidencia de 1.8 millones de casos en 2018 según datos de GLOBO$\mathrm{CAN}^{1,2}$. Las metástasis tiroideas son tremendamente infrecuentes, situándose su incidencia en torno al 1-3\% según los diferentes datos. El cáncer de colon es el segundo tumor que con más frecuencia produce metástasis en este órgano, después del cáncer renal ${ }^{3}$. Aunque la mayoría de los pacientes que presentan metástasis tiroideas se diagnostican en el contexto de un cáncer previo conocido, se han descrito casos de metástasis tiroideas en pacientes con un tumor

\footnotetext{
Correspondencia:

*Patricia Cruz-Castellanos

Paseo de la Castellana 264

Fecha de recepción: 30-04-2021

C.P. 28046, Madrid, España

E-mail: patriciacruzcastellanos@gmail.com

Fecha de aceptación: 10-05-2021

DOI: $10.24875 / C I R U .21000401$

0009-7411/@ 2021 Academia Mexicana de Cirugía. Publicado por Permanyer. Este es un artículo open access bajo la licencia CC BY-NC-ND (http://creativecommons.org/licenses/by-nc-nd/4.0/).
} 
primario oculto. La exploración física y los estudios de imagen son importantes para el diagnóstico de las metástasis tiroideas. Su manejo terapéutico es controvertido, dada la escasez de datos. La mayoría de los pacientes que presentan metástasis tiroideas serán tratados con intención paliativa. Sin embargo, en pacientes seleccionados se puede realizar trata miento quirúrgico mediante lobectomía o tiroidectomía total ${ }^{4}$.

En este caso clínico exponemos la aparición de una metástasis tiroidea en una paciente con cáncer de colon metastásico de larga evolución. Además, presentamos los resultados del estudio de secuenciación masiva que se realizó a la paciente.

\section{Caso clínico}

Mujer de 55 años diagnosticada de adenocarcinoma de sigma KRAS no mutado con estadio clínico cT3NxM1 por afectación hepática única. Dada la posibilidad de resecabilidad de la lesión hepática se consideró a la paciente como oligometastásica, por lo que se realizaron hepatectomía derecha y posterior sigmoidectomía. Tras ambas intervenciones, la paciente recibió tratamiento adyuvante basado en capecitabina y oxaliplatino, e inició revisiones. Tras 8 meses, en la tomografía computarizada (TC) toraco-abdomino-pélvica de control apareció una lesión pulmonar única localizada en el lóbulo superior derecho. El estudio se completó con una tomografía por emisión de positrones (PET-TC) que descartó la presencia de otro foco de enfermedad. Dado que la lesión era inaccesible para realizar una punción-aspiración con aguja fina (PAAF), se optó por cirugía directa, realizándose lobectomía superior derecha sin complicaciones y con resultado anatomopatológico de metástasis pulmonar de adenocarcinoma enteroide pobremente diferenciado. Después de la cirugía se optó por la administración de otros cuatro ciclos de oxaliplatino y capecitabina como consolidación. Tras 2 años de seguimiento sin incidencias, en la TC de control apareció una lesión suprarrenal izquierda de $33 \times 15 \mathrm{~mm}$. La PET-TC confirmó la presencia de enfermedad en esta localización y además mostró hipercaptación a nivel del mediastino sugestiva de recaída. En esta situación se optó por iniciar tratamiento sistémico introduciendo, junto con la quimioterapia, un antiangiogénico. Se administraron en total seis ciclos, con muy buena respuesta, dism inuyendo la captación a nivel del mediastino y el tamaño de la lesión suprarrenal. En esta situación, se presentó el caso en el comité multidisciplinario y, tras considerar la larga evolución de la enfermedad y la baja carga tumoral, se decidió administrar radioterapia a dosis radical sobre la recaída mediastínica e intervención de la lesión suprarrenal, iniciando posteriormente revisiones. Con un intervalo libre de enfermedad de 2 años, la paciente presentó una nueva recaída en el lecho quirúrgico de la suprarrenalectomía y la aparición de un nódulo sospechoso a nivel tiroideo, con hipercaptación en la PET-TC. Por lo excepcional del caso, antes de plantear opciones terapéuticas se realizó una PAAF de la lesión tiroidea, que fue compatible con metástasis de adenocarcinoma enteroide pobremente diferenciado. En esta situación, dada la presencia de dos focos de enfermedad activos, se decidió iniciar tratamiento con esquema de quimioterapia y anti-EGFR (epidermal growth factor receptor). Tras 6 meses de tratamiento, una nueva PET-TC mostró una respuesta completa de la lesión tiroidea y parcial de la suprarrenal, optándose entonces por continuar con mantenimiento con anti-EGFR y administración de radioterapia local a nivel suprarrenal, con lo que se consiguió de nuevo un control óptimo de la enfermedad. Sin embargo, tras 12 meses de mantenimiento con anti-EGFR la paciente presentó nueva progresión, en esta ocasión de localización hepática, y se iniciaron varios tratamientos sin éxito. Debido a la larga evolución de la enfermedad y el patrón metastásico atípico, se decidió ampliar el estudio molecular para obtener una mejor caracterización del tumor y valorar posibles dianas terapéuticas. Para ello, se realizó un panel de secuenciación masiva (NGS) de la pieza tumoral, identificándose alteraciones genómicas en APC (R232), ERRF1 (R244) y TP53 (G266V), pero ninguna de ellas con opciones terapéuticas reales. Además, los genes ACVR1B, ERRB4, MSH6, NOTCH3 y TNFRSF14 mostraron variantes de significado incierto. La carga mutacional (TMB) se informó como $6.30 \mathrm{Mb}$ y se descartó inestabilidad de microsatélites (MSI), por lo que la paciente no pudo optar a tratamiento con inmunoterapia. En esta situación, tras 13 años de evolución se suspendió el tratamiento activo dadas las pocas opciones terapéuticas y la falta de respuesta a la quimioterapia convencional.

\section{Discusión}

En este caso clínico exponemos un cáncer de coIon de larga evolución con aparición de metástasis tiroidea. La glándula tiroides es un órgano altamente 
vascularizado, donde la afectación metastásica es excepcional, por lo que clásicamente se ha considerado como un órgano "santuario". Las diferentes series de casos sitúan la incidencia de las metástasis en esta localización en torno al 1-3\%, aumentando hasta el $25 \%$ en series que incluyen necropsias ${ }^{3,4}$. Según los datos publicados en la literatura, los tumores con mayor potencial de diseminación metastásica a la glándula tiroides son los cánceres renal (48.1\%), colorrectal (10.1\%), de pulmón $(8.3 \%$ ) y de mama (7.8\%).

Actualmente se desconoce el motivo por el que las metástasis son tan infrecuentes en la glándula tiroides a pesar de su alta vascularización, pero se postulan dos posibles teorías. La primera de ellas se basa en la propia cinética del cuerpo: dado el alto flujo de sangre que presenta esta glándula, su paso por ella es muy rápido y esto impediría la posibilidad de anidar a las células tumorales (teoría mecánica). La segunda teoría plantea que la presencia natural de yodo en la glándula tiroides impediría el crecimiento tumoral (teoría metabólica). Desde el punto de vista epidemiológico, estas metástasis parecen ser más frecuentes en las mujeres que en los hombres (ratio 1.4:1) y en tiroides de morfología nodular (44.2\%). La edad media de presentación es a los 60 años. Se estiman una media de 69.9 meses y una mediana de 53 meses entre el diagnóstico del tumor primario y la aparición de la lesión tiroidea, aunque hasta en un $20 \%$ de los casos la metástasis tiroidea puede aparecer de forma sincrónica con el tumor primario ${ }^{3}$. Clínicamente, lo más frecuente es que se detecten de manera incidental al realizar una prueba de imagen, como ocurrió en nuestra paciente, siendo muy infrecuente su debut por la clínica acompañante. De hecho, en los últimos años, debido a la incorporación de la PET-TC en la estadificación y el seguimiento de los pacientes oncológicos, su incidencia ha aumentado ligeramente. De forma excepcional pueden dar síntomas por compresión local (si se trata de una lesión de gran tamaño) o alteraciones endocrinológicas (hipotiroidismo o hipertiroidismo) debido a la presencia de émbolos tumorales que desencadenarían daño glandular y vertido masivo de hormonas a la sangre. En cuanto a su diagnóstico, ante la aparición de un nódulo tiroideo sospechoso en un paciente oncológico es necesario determinar su naturaleza primaria o metastásica. Para ello, lo más habitual es realizar una PAAF guiada por ecografía. La citología resultante debería orientar sobre el diagnóstico, pero en ocasiones es difícil diferenciar una lesión pri- maria de una secundaria, sobre todo en tumores indiferenciados y anaplásicos ${ }^{5}$.

Su manejo es controvertido, sin que existan unas directrices claras. Para valorar el mejor abordaje se deben tener en cuenta la situación oncológica basal del paciente, el tipo de tumor primario y la presencia o no de síntomas acompañantes. La cirugía mediante tiroidectomía total con o sin linfadenectomía cervical se puede plantear ante una lesión única, siempre que el tumor primario se encuentre controlado o en remisión. Si por el contrario, como en nuestro caso, existiesen más focos de enfermedad activa, se debería descartar la cirugía, salvo que se tratase de un procedimiento paliativo, y optar por el tratamiento sistémico. Un hecho importante es que la sensibilidad a la quimioterapia de las lesiones tiroideas es baja, y además su aparición se ha considerado que es un signo de mal pronóstico ${ }^{6}$. Este hecho contrasta con nuestra experiencia, pues con la combinación de quimioterapia y anti-EGFR se consiguió una respuesta completa verificada por TC y PET-TC. Por este motivo, y debido a la evolución peculiar del cáncer de colon, se decidió ampliar el estudio histológico mediante una NGS con el objetivo de obtener un perfil molecular e identificar alguna diana molecular tratable. Se analizaron 324 genes, evaluando las cuatro principales alteraciones genómicas (sustitución de bases, deleciones e inserciones, alteración del número de copias y reordenamientos), así como la TMB y la MSI. Como se ha mencionado previamente, se identificaron alteraciones en APC (R232), ERRF1 (R244) y TP53 (G266V), sin que en la actualidad exista ningún tratamiento válido para ellas. Tanto el gen APC como el TP53 han sido ampliamente estudiados en el cáncer de colon, y actualmente se sabe que la mutación somática de APC ocurre de forma precoz en la tumorogénesis, mientras que la de TP53 es más tardía. Sin embargo, su presencia no se ha asociado a mejor 0 peor pronóstico en esta patología, ni a patrones metastásicos determinados ${ }^{7}$. Por otro lado, la determinación de la TMB y la MSI ha cobrado importancia en los últimos años debido a la posibilidad de tratamiento con inmunoterapia. Actualmente, la TMB se posiciona como un posible biomarcador para el uso de inhibidores de PD-1/PDL-1, con independencia de la histología del tumor primario. El ensayo clínico de fase II KEYNOTE-158, en el que se incluyeron pacientes con distintas histologías, demostró un aumento de la supervivencia libre de progresión con el uso de inmunoterapia ante la presencia de una TMB alta $(>10$ mutaciones/megabase). Un aspecto controvertido es 
que en este estudio no se incluyeron todas las histologías, faltando por ejemplo el cáncer de mama y el de colon, por lo que actualmente hay que tener prudencia en la extrapolación de los datos ${ }^{8}$. Además, resultados recientes en cáncer de colon parecen sugerir una baja tasa de respuesta a la inmunoterapia a pesar de una TMB alta. En relación a la MSI, la presencia de inestabilidad de microsatélites (MSI-H) también se ha asociado a buena respuesta a la inmunoterapia. Estos datos han llevado a la aprobación del pembrolizumab en monoterapia con independencia de la histología del tumor primario, tratándose de la primera aprobación agnóstica de la historia ${ }^{9}$.

Con este caso queremos destacar la importancia del manejo multidisciplinario en el cáncer de colon, sobre todos en aquellos pacientes de larga evolución, debido a la aparición de metástasis únicas a lo largo de su enfermedad. Además, resaltamos la excepcionalidad de las lesiones tiroideas secundarias a cáncer de colon. Por último, también hay que destacar la importancia de realizar un perfil molecular a los pacientes con evolución anómala para la mejor tipificación y la identificación de posibles dianas terapéuticas en el marco de la medicina personalizada.

\section{Agradecimientos}

Los autores agradecen a los familiares y a los pacientes.

\section{Financiamiento}

Los autores declaran no haber recibido financiamiento para este estudio.

\section{Conflicto de intereses}

Los autores declaran no tener conflicto de intereses.

\section{Responsabilidades éticas}

Protección de personas y animales. Los autores declaran que para esta investigación no se han realizado experimentos en seres humanos ni en animales.

Confidencialidad de los datos. Los autores declaran que han seguido los protocolos de su centro de trabajo sobre la publicación de datos de pacientes.

Derecho a la privacidad y consentimiento informado. Los autores han obtenido el consentimiento informado de los pacientes y/o sujetos referidos en el artículo. Este documento obra en poder del autor de correspondencia.

\section{Bibliografía}

1. Henley SJ, Ward ME, Scott S, Ma J, Anderson RN, Firth AU, et al. Annua report to the nation on the status of cancer, part I: national cancer statistics. Cancer. 2020;126:2225.

2. Siegel RL, Miller KD, Fuchs HE, Jemal A. Cancer statistics, 2021. CA Cancer J Clin. 2021;71:7.

3. Chung AY, Tran TB, Brumund KT, Weisman RA, Bouvet M. Metastases to the thyroid: a review of the literature from the last decade. Thyroid. 2012;22:258-68.

4. Cichon S, Anielski R, Konturek A, Barczynski M, Cichon W. Metastases to the thyroid gland: seventeen cases operated on in a single clinical center. Langenbecks Arch Surg. 2006;391:581-7.

6. Kim TY, Kim WB, Gong G, Hong SJ, Shong YK. Metastasis to the thyroid diagnosed by fine-needle aspiration biopsy. Clin Endocrinol (Oxford). 2005;62:236-41.

7. Cheung WY, Brierley J, Mackay HJ. Treatment of rectal cancer metastases to the thyroid gland: report of two cases. Clin Colorectal Cancer. 2008;7:280-2.

8. Cancer Genome Atlas Network. Comprehensive molecular characterization of human colon and rectal cancer. Nature. 2012;487:330-7.

9. Marabelle A, Fakih M, Lopez J, Shah M, Shapira-Frommer R, Nakagawa K, et al. Association of tumour mutational burden with outcomes in patients with advanced solid tumours treated with pembrolizumab: prospective biomarker analysis of the multicohort, open-label, phase 2 KEYNOTE-158 study. Lancet Oncol. 2020;21:1353-65. 